\title{
Effect of Sulfonation Level on Lignin/Carbon Composite Electrodes for Large-Scale Organic Batteries
}

\author{
Ujwala Ail, Jaywant Phopase, Jakob Nilsson, Zia Ullah Khan, Olle Inganäs, Magnus Berggren, \\ and Xavier Crispin*
}

Cite This: ACS Sustainable Chem. Eng. 2020, 8, 17933-17944

Read Online

\section{ACCESS |}

山l Metrics \& More

Article Recommendations

Supporting Information

ABSTRACT: The key figure-of-merit for materials in stationary energy storage applications, such as large-scale energy storage for buildings and grids, is the cost per kilo per electrochemical cycle, rather than the energy density. In this regard, forest-based biopolymers such as lignin, are attractive, as they are abundant on Earth. Here, we explored lignin as an electroactive battery material, able to store two electrons per hydroquinone aromatic ring, with the targeted operation in aqueous electrolytes. The impact of the sulfonation level of lignin on the performance of its composite electrode with carbon was investigated by considering three lignin derivatives: lignosulfonate (LS), partially desulfonated lignosulfonate (DSLS), and fully

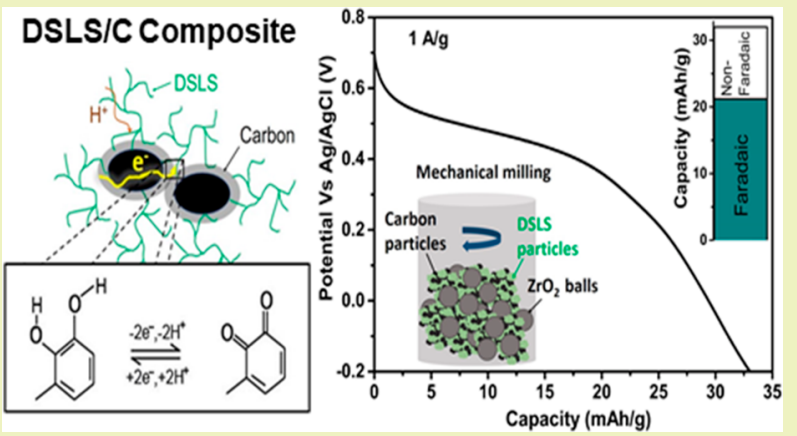
desulfonated lignin (KL, lignin produced by the kraft process). Partial desulfonation helped in better stability of the composite in aqueous media, simultaneously favoring its water processability. In this way, a route to promote ionic conductivity within the lignin/carbon composite electrodes was developed, facilitating the access to the entire bulk of the volumetric electrodes. Electrochemical performance of DSLS/C showed highly dominant Faradaic contribution $(66 \%)$ towards the total capacity, indicating an efficient mixed ionic-electronic transport within the lignin-carbon phase, displaying a capacity of $38 \mathrm{mAh} / \mathrm{g}$ at $0.25 \mathrm{~A} / \mathrm{g}$ and $69 \%$ of capacity retention after $2200 \mathrm{cycles}$ at a rate of $1 \mathrm{~A} / \mathrm{g}$.

KEYWORDS: Lignin, Lignosulfonate, Carbon black, Supercapacitor, Battery, Ball milling, Nanocomposite, Electrode

\section{INTRODUCTION}

Integration of renewable energy sources into our day-to-day lives is essential to reduce the dependence on the conventional energy technologies based on fossil fuels. Large-scale energy storage systems connected to the grid are one of the main components in this transition and context, as they ensure the reliability and flexibility of the renewable energy systems by damping the power fluctuations of intermittent solar and wind power energy sources. In this regard, the ability to store and distribute electricity in sustainable batteries, based on noncritical raw materials that last a long time, is transformative for the energy sector in our society. ${ }^{1,2}$ Energy storage specifications of a battery are related to the specific battery technology and the amount of electroactive material included. Hence, a major constraint for the electroactive material to be used in large-scale applications, such as in energy-grids, is its abundance on Earth related to the cost of deriving a material with desired performance parameters. Thus, the economic viability of a technology is related not only to the performance of the materials with considerations of cost, but also to the budget of the manufacturing techniques and their compatibility with available technologies and industrial processes, as well as their environmental impacts. ${ }^{3}$
In this regard, biobased polymers such as lignin, originating from the forest and agricultural feed-stocks, represent a very attractive choice to explore. Chemically, lignin is composed of amorphous, heterogeneous, and highly cross-linked polymers of phenolic nature. A large amount of lignin is obtained as a byproduct of industrial processing of cellulose pulp and production of cellulosic fuels. ${ }^{4}$ The dominant lignin isolation processes used today are the sulfite and kraft processes producing lignosulfonate (LS) and kraft lignin (KL), respectively. Unlike the lignosulfonate, kraft lignin is insoluble in water at neutral $\mathrm{pH}$, which limits its industrial applications. LSs generally have a higher degree of sulfonation than that of KL. The sulfonate group makes LS anionically charged and facilitates its water solubility. ${ }^{5}$ In addition to sulfonic acid groups, it also has hydrophilic functional groups such as hydroxyl, phenol, and carboxylic acid (Figure 1a). This important characteristic of being water processable makes LS

Received: July 22, 2020

Revised: October 6, 2020

Published: November 30, 2020 
(a)

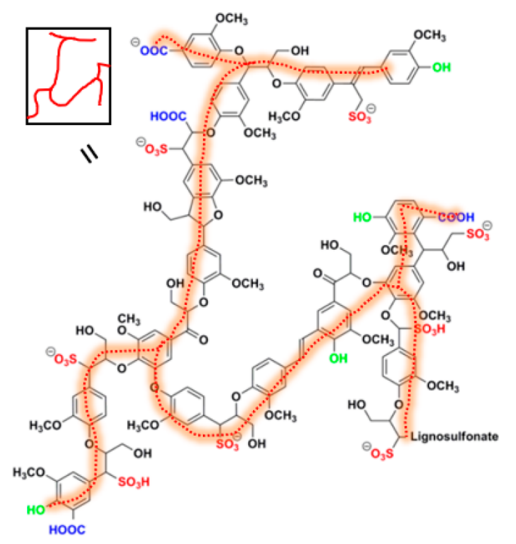

(b)

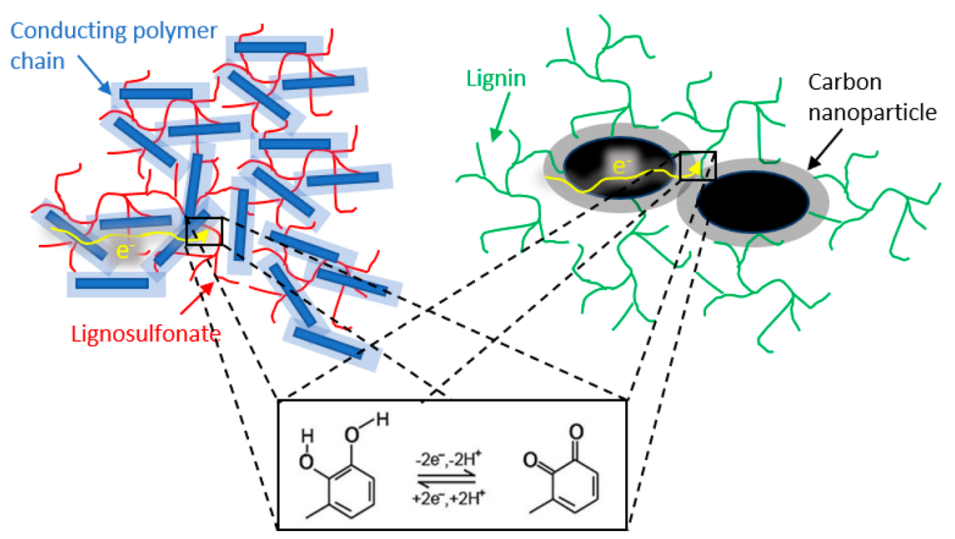

Figure 1. (a) Chemical structure of LS, (b) sketch of the LS-conducting polymer composite, where the electrons can pass through the composite by hopping between adjacent conducting polymer chains (dark blue rectangles) and reach the redox quinone site on the LS (red color lines). The area reachable by the electron is illustrated as light blue color area around the polymer chains, which when overlapping with another polymer chains ensure transport or overlapping with LS ensure its local redox activity. The bottom inset illustrates the redox reaction of lignin. (c) Sketch of the kraft lignin-carbon nanoparticle composite displaying a much lower overlap between the area reachable by the electron and lignin. Only this interfacial volume would display the electrochemical redox process of lignin.

a safe material and the most explored form of lignin in commercial applications. In the perspective of energy storage, lignin has a large amount of catechol groups that can be electrochemically oxidized and reduced in two-electron processes. In aqueous acidic solutions, quinone/hydroquinone couples generally provide a reversible two-electron/two-proton redox reaction $\left(2 \mathrm{e}^{-} / 2 \mathrm{H}^{+}\right)$(Figure $1 \mathrm{~b}-\mathrm{c}$ ). Since lignin is nonconductive, an electrical conductor is required, as a conductive matrix in the lignin-conductor composite, to bring the electronic charge carriers to the quinone redox sites of lignin and enable charge storage inside the lignin phase. In this direction, conductors such as conducting polymers, ${ }^{6-9}$ graphene, ${ }^{10}$ partially reduced graphene oxide, ${ }^{11}$ carbon nanotube, $^{12}$ partially reduced graphite oxide, ${ }^{13}$ graphite, ${ }^{14}$ and conductive carbon ${ }^{15,16}$ have been composited with KL or LS.

Until now, two forms of lignin derivatives have been mostly studied, LS and KL. Even though the water solubility of LS makes it easily processable for large-scale energy storage devices, ${ }^{17}$ it is also one of the main drawbacks as it makes the LS phase leak out from the electrode composite into the electrolyte. This typically leads to a drastic drop in performance under electrochemical cycling. ${ }^{18}$ Part of the solubility problem is solved by directly complexing the negatively charged LS electrostatically to the positively charged conducting polymer. However, for some conducting polymers, upon cathodic bias, the conducting polymer becomes neutral, which liberates LS to leak out into the electrolyte. So, the stability of a LS-conducting polymer composite is good but might have its limitation at some cathodic potentials. The advantage of such composites is the intimate mixing of the conductor with LS at the molecular scale so that most of the redox quinone groups of lignin become accessible with this polymeric conductor, thus leading to a high specific capacity $(72-80 \mathrm{mAh} / \mathrm{g} \text { at } 1 \mathrm{~A} / \mathrm{g})^{8,9}$ (Figure $1 \mathrm{~b}$ ). The molecular intimacy of this interaction was illustrated when LS in solution could be ion-exchanged with a small counteranion contained in a positively charged polymer to form a polymer-LS nanocomposite. ${ }^{17}$ Another development pathway is to consider insoluble kraft lignin, which is not possible to complex with charged conducting polymers. Instead, various carbon-based conductors have been utilized. Here, the advantage is that the lignin is not soluble in the electrolyte, which improves the stability of the composite. However, the Faradaic contribution from the redox process of lignin is relatively lower than that of the lignin-conducting polymer composite. Indeed, providing electrical conductivity at the molecular scale within lignin is not trivial, since carbon nanoparticles are much larger and provide less favorable entropy of mixing while comparing with the low-dimensional conducting polymer chains. Because of the large size of the carbon nanoparticles and the electrically insulating property of lignin, only the carbon-lignin interface becomes electroactive and is involved in the active charge storage (Figure 1c). The strategy is thus to intermix carbon and lignin as much as possible to increase this interfacial volume. An interesting track is for instance the preparation of $\mathrm{KL}$ and carbon composites using oscillatory milling with the purpose of breaking the carbon particles into smaller components which leads to a better closeness of the carbon conducting phase with the lignin. ${ }^{14,15}$

Finally, worth mentioning is the observation that the charge storage capacity of the $\mathrm{KL} /$ carbon composite was influenced significantly by the KL interfacial thickness, probably due to the ionic diffusion limitations. ${ }^{15}$ Hence, a higher KL content in the composite was unable to ensure a higher Faradaic capacity as $\mathrm{KL}$ alone does not conduct ions, and both electrons and ions are necessary for the efficient redox reactions (while LS transports ions). Hence, for battery applications, this is a key issue, wherein thick films are desired.

In this work, we investigate the role of the sulfonation level of lignin on its ionic conductivity and solubility with the goal to see if an intermediate sulfonation level can combine ionic conductivity and water insolubility. In our approach, mechanical milling by a planetary mill has been employed to achieve intimate electrical contact between the desulfonated LS (DSLS) and conductive carbon black, followed by the water-based binder to prepare the composite electrode material. We compare the properties of DSLS with two extremes, namely, LS (completely soluble in water) and kraft lignin $(\mathrm{KL})$, via a lignoboost process (insoluble in water). 
(a)

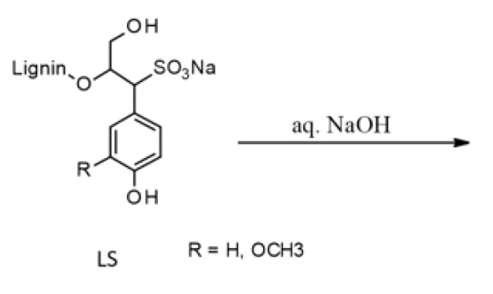

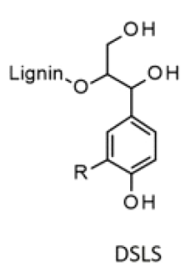

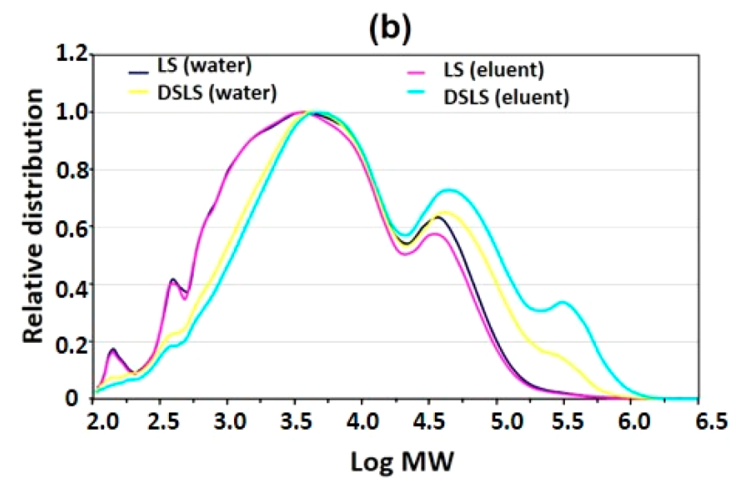

Figure 2. (a) Scheme of desulfonation of LS, (b) relative distribution of molecular weight versus logarithmic molecular weight for LS and DSLS in water and eluent.

\section{EXPERIMENTAL SECTION}

Procedure for the Desulfonation of LS. Desulfonation of LS was carried out via hydrolysis of sulfonic acid groups. For this purpose, $50 \mathrm{~g}$ of LS powder (supplied by Domsjö) and $8 \mathrm{~g}$ of $\mathrm{NaOH}$ (Sigma-Aldrich) were dissolved in $300 \mathrm{~mL}$ of distilled water, followed by refluxing for $5 \mathrm{~h}$ in an oil bath. After this, the solution was cooled to $90{ }^{\circ} \mathrm{C}$, and $100 \mathrm{~mL}$ of $60 \% \mathrm{H}_{2} \mathrm{SO}_{4}$ was added to the reaction mixture while stirring at room temperature for $2 \mathrm{~h}$. Subsequently, the product, DSLS, was collected by centrifugation, washed two times with distilled water, and dried in a vacuum oven at $40^{\circ} \mathrm{C}$. Figure $2 \mathrm{a}, \mathrm{b}$ shows the scheme of the desulfonation process and the molecular weight distribution. Note that sulfuric acid was used as a neutralization agent for the alkaline reaction mixture. There are two reasons for that. First, the sulfate ion is non-nucleophilic and cannot react with lignin in diluted form. Second, the lignosulfonate already contains some sodium sulfate as residue from the pulp and paper process. When comparing different lignin fractions, we prefer to minimize the different types of contaminations in the sample even though the analysis method becomes a bit more complex with varying sodium sulfate content in the sample.

DS-LS Material Characterization. Gel Permeation Chromatography (GPC). Size exclusion chromatography with UV detection at $256 \mathrm{~nm}$ was carried out to estimate the change in molecular weight of DSLS. For this purpose, LS and DSLS were dissolved in water and in eluent (sodium borate buffer $\mathrm{pH} 9+10 \% \mathrm{MeOH}$ ) and filtered using a $0.45 \mu \mathrm{m}$ syringe filter before injection. Since the DSLS sample was only partially soluble in water, the insoluble fraction was removed by filtration. Calibration was carried out using polystyrenesulfonate with known molecular weight $\left(M_{\mathrm{w}}\right)$ varying from $122 \mathrm{~g} / \mathrm{mol}$ to $679000 \mathrm{~g} /$ mol, and measurement was performed at $25{ }^{\circ} \mathrm{C}$. Columns SB-802.5 HQ, SB-803 HQ and SB-804 HQ (Shodex) were used for HPLCanalysis.

Figure $2 \mathrm{~b}$ shows the relative distribution and molecular weight of the LS and DSLS samples dissolved in both water and borate eluent. There is a large difference in molecular weight for the DSLS and the starting material LS. Molecular weight of LS is $11.4 \mathrm{kDa}$ while for DSLS is $41 \mathrm{kDa}$. A lot of the low molecular weight materials including sugars are washed away in the washing procedure after the neutralization of the lignin. In addition, DSLS also contains a lot of high molecular weight lignin with $M_{\mathrm{w}}$ of several $100 \mathrm{kDa}$. These fractions were not present before our chemical treatment and have formed due to condensation reactions. The molecular weight distributions are summarized in Table 1.

Elementary Analysis. Elementary analysis was carried out to determine the total sulfur $\left(S_{\text {tot }}\right)$ content, and the sulfate content $\left(\mathrm{S}_{\mathrm{SO}_{4}}\right)$ was measured by titration. The sulfonic acid $\left(S_{\mathrm{SO}_{3}}\right)$ content was assumed to be $S_{\mathrm{SO}_{3}}=S_{\text {tot }}-S_{\mathrm{SO}_{4}}$. The sulfonate content was reduced by $20 \%$ after desulfonation, as shown in Table 2.

Preparation of DSLS/Carbon Composite Electrode. The electrode nanocomposite material has been fabricated via solvent-free mechanical milling (ball milling) of conductive carbon (ENSACO

\section{Table 1. Detailed Information about the Molecular Weight ${ }^{a}$}

\begin{tabular}{lcccc}
\multicolumn{1}{c}{ sample } & $M_{\mathrm{W}}$ & $\log M_{\mathrm{w}}$ & $M_{\mathrm{n}}$ & $M_{\mathrm{p}}$ \\
LS in water & 12700 & 4.1 & 1070 & 3760 \\
LS in eluent & 11400 & 4.1 & 1050 & 3440 \\
DSLS water & 23700 & 4.4 & 1410 & 4190 \\
DSLS in eluent & 41400 & 4.6 & 1730 & 4570
\end{tabular}

${ }^{a} M_{\mathrm{w}}=$ Weight-averaged molecular weight. $M_{\mathrm{n}}=$ Number-averaged molecular weight. $M_{\mathrm{p}}=$ Peak molecular weight.

Table 2. Sulfur Content in LS and DSLS

\begin{tabular}{ccc} 
sulfur source & LS & DSLS \\
$S_{\text {tot }}(\%)$ & 7.67 & 9.39 \\
$S_{\mathrm{SO}_{4}}(\%)$ & 1.95 & 4.60 \\
$S_{\mathrm{SO}_{3}}(\%)$ & 5.72 & 4.79 \\
\hline
\end{tabular}

360 G from IMERYS Graphite and Carbon, with BET surface area of $780 \mathrm{~m}^{2} / \mathrm{g}$ ) and desulfonated LS (DSLS), in a planetary ball mill, Retsch PM 100 using $\mathrm{ZrO}_{2}$ milling media. The dry ratio of the DSLS and carbon was 1:1. The milled powder was then combined with $6 \mathrm{wt}$ $\%$ of CMC/SBR (carboxymethylated cellulose/styrene butadiene rubber) binder system, and the resulting slurry was applied onto the carbon coated SS (stainless steel) electrodes. Graphite (flakes, mp 3652-3697 C, density $1.9 \mathrm{gcm}^{-3}$, Sigma-Aldrich) was used for comparison of carbon type in our process.

A microstructural study was carried out using scanning electron microscopy (SEM, Zeiss Sigma 500 Gemini). The contact angle of the water on the lignin samples was measured using an optical contact angle and surface tension meter (KSV Instruments CAM 200). The layers formed for the contact angle analysis were obtained by depositing the water solution/slurry of the different lignin samples on a glass substrate and drying at $70{ }^{\circ} \mathrm{C}$ for $15 \mathrm{~min}$. Impedance measurements were carried out using an impedance spectrometer (an Alpha high-resolution dielectric analyzer, Novocontrol Technologies $\mathrm{GmbH}$ ) using a two-point probe method. An AC voltage of $5 \mathrm{mV}$ is applied while sweeping the frequency from $1 \mathrm{MHz}$ to $1 \mathrm{~Hz}$, and the humidity was maintained at $90 \% \mathrm{RH}$ and $60 \% \mathrm{RH}$. The layers were made by drying from a drop-casted water solution/slurry of lignin between the two Au lateral electrodes, and those were connected to the impedance spectrometer. PerkinElmer Lambda 900 was used for the UV-visible spectroscopy of the samples in the wavelength region of $250-700 \mathrm{~nm}$.

Electrochemical Characterization. Electrochemical performance of the electrodes was studied using a computer-controlled potentiostat (SP200, BioLogic). The electrodes were evaluated by the cyclic voltammetry $(\mathrm{CV})$ at different scan rates and the galvanostatic charge-discharge (GCD) by applying different charging rates (mass normalized current) in a three-electrode system under nitrogen atmosphere. The measurements were also carried out in ambient 

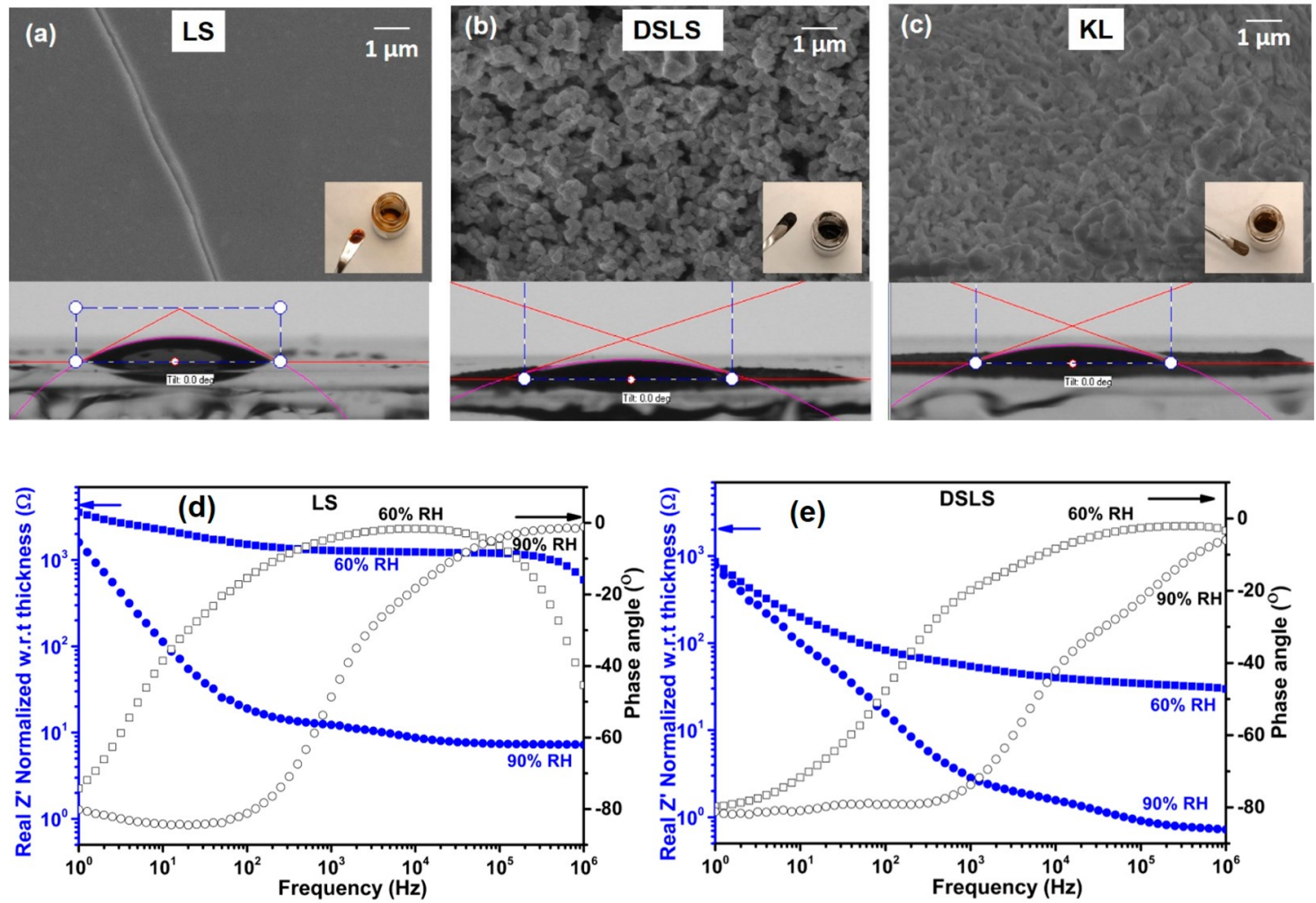

Figure 3. SEM images and contact angle of a water drop for (a) LS layer, (b) DSLS layer, and (c) KL layer. The inset picture illustrates the water solution/slurry of different types of lignin samples. Bode plots of LS (d) and DSLS layers (e) at different relative humidity values.

conditions. There are no significant changes in the electrochemical activity in both the atmospheres in the potential ranges used in the present study. A Pt mesh was used as a counter electrode, potential was measured versus the $\mathrm{Ag} / \mathrm{AgCl}$ reference electrode, and the electrolyte was $0.1 \mathrm{M} \mathrm{HClO}_{4}$. The charge capacity $(\mathrm{mAh} / \mathrm{g})$ was obtained by dividing the product of the discharge time (in $\mathrm{s}$ ) and the discharge current (in $\mathrm{mA}$ ) by the product of 3600 and mass of the total electrode material (in g).

\section{RESULTS}

Characterization of Lignin with Various Sulfonation Levels. We begin by investigating the difference between the three types of lignins, namely, LS, partially desulfonated LS (i.e., DSLS), and completely desulfonated KL. For this purpose, each lignin sample was immersed into water. In the case of LS, the product is a solution, whereas for DSLS and KL, the product is an aqueous slurry. It is important to note that even though KL does not dissolve in water, it is wetted by water due to the presence of a lot of hydrophilic functional groups such as hydroxyl, phenol, and carboxylic acid. A similar observation is also seen in DSLS. Figure 3a shows the SEM micrograph of different lignin layers that were prepared by the above-mentioned method followed by coating on conducting substrates for the SEM study. The DSLS layer obtained by drying the slurry shows a porous surface with well-defined grain morphology formed by the solid particles from the slurry. This morphology is totally different than the smooth solid polymer film made from the LS solution. The microstructure of the KL layer has some similarity with the DSLS layer. However, the particles in the KL slurry seem to have coalesced with each other leading to a more compact, thus much less porous morphology compared to the DSLS. The grains are in the range of $100 \mathrm{~nm}$. These microstructural variations of the three different lignin layers are expected to also have an impact on the wetting behavior of water in addition to their sulfonation level. To illustrate the water affinity for wetting of the three lignin layers, the contact angles $\Phi$ of a water drop is measured on the surface of the LS $\left(\Phi=28^{\circ}\right)$, DSLS ( $\Phi=$ $\left.18^{\circ}\right)$, and $\mathrm{KL}\left(\Phi=20^{\circ}\right)$ layers (Figure $\left.3 \mathrm{~b}\right)$. The contact angles on DSLS and $\mathrm{KL}$ were reduced to below $10^{\circ}$ within $2 \mathrm{~s}$ due to their microporous structures and capillary forces absorbing water in the pores. The wetting angle of LS varies also dynamically and reduces to $21^{\circ}$ in $1 \mathrm{~min}$, most likely due to a slow solubility of LS in the water drop.

These comparisons between the lignin layers illustrate the impact of the concentration of sulfonate groups on the physicochemical properties of the lignins. This enables us to identify which of the lignins should be used to make the electrode for a battery operating in an aqueous electrolyte since one of our target is to consider nonflammable aqueous electrolytes for large-scale battery applications. In that context, the interaction of the lignin layers with water is important. The catechol-type groups in the lignins can undergo a redox reaction and thus store electricity (two electrons per catechol group) (see Figure 1); which is the motivation why lignin can be used as true energy storage material. But for that purpose, electrons and ions (protons) need to reach the catechol groups. The lignin cannot transport electrons and that is why lignin will subsequently be composited with carbon nanoparticles, which is an electronic conductor. But it is thus crucial to understand which of the lignin layers could lead to a better ionic conductivity. For this reason, we further studied the ionic conductivity of these layers by impedance spectroscopy measured at $60 \% \mathrm{RH}$ and $90 \% \mathrm{RH}$. In LS, the anions and sulfonate are immobile and covalently attached to the 

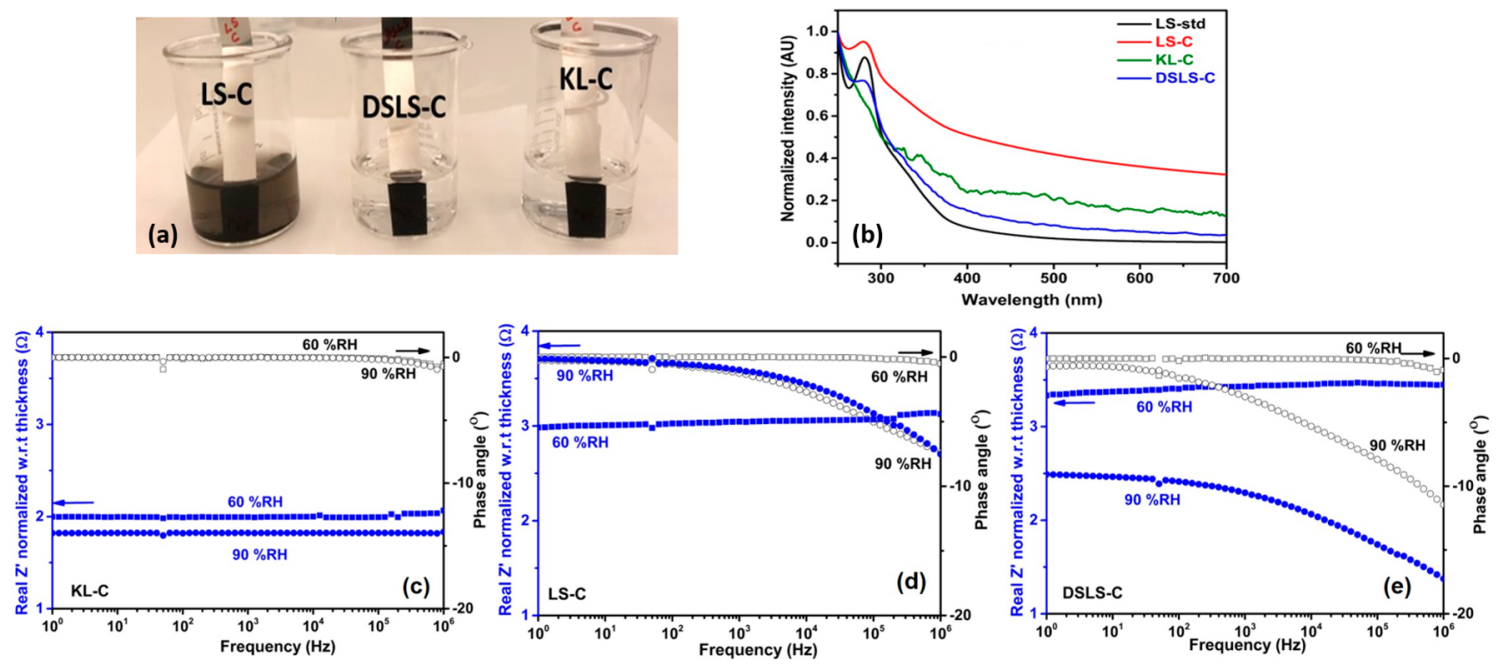

Figure 4. (a) Composite electrodes dipped in water for $2 \mathrm{~h}$, (b) the UV-vis spectra of the water after $2 \mathrm{~h}$ of dipping the composite electrodes, (ce) the Bode plots at different relative humidity values for KL/C, LS/C, and DSLS/C samples, respectively.

branched biopolymer, while the positively charged counterions $\left(\mathrm{H}^{+}\right.$or $\left.\mathrm{Na}^{+}\right)$give rise to ionic conductivity, as well as residual sulfate ions coming from the synthesis. Typically in polyelectrolytes, the conductivity of ions increase exponentially with the humidity until it reaches a plateau at high humidity $(\sim 70-80 \% \mathrm{RH})$ where percolation water channels are formed inside and on the surface of the materials. ${ }^{19,20}$ Hence, the ionic conductivity at $60 \% \mathrm{RH}$ is dominated by the material property to transport ions, while at $90 \% \mathrm{RH}$ it is dominated by the transport of ions in water percolation paths, thus approaching liquid electrolyte and simulating the electrode in contact with a liquid electrolyte in a battery. AC probe voltage of $5 \mathrm{mV}$ was used, and its frequency was swept from $1 \mathrm{MHz}$ to $1 \mathrm{~Hz}$. The first observation is that it was not possible to characterize the KL layer by impedance spectroscopy simply because it was too insulating both for electrons and ions $\left(\sim 10^{9} \Omega\right)$. On the contrary, the impedance spectrum could be measured both for LS and DSLS. The Bode plot is displayed in Figure 3d,e for LS and DSLS respectively. Bode plots allow one to understand and separate the ionic mechanisms in the time/frequency domain, mainly the oscillation of the ions in the AC field, which is a resistive character (phase angle $\Phi=0^{\circ}$ ) from which the ionic conductivity can be extracted. In the case of LS (Figure 3d), at 60\% RH, three different regimes are found as in other sulfonated polyelectrolytes, ${ }^{19}$ namely, dipolar relaxation $(>1 \mathrm{M} \mathrm{Hz})$, ionic migration $(40 \mathrm{kHz}-3 \mathrm{kHz}$, indicated by $\Phi=$ $\left.0^{\circ}\right)$, and the accumulation of ions at the electrode surface forming the electric double layer capacitor (phase angle $\Phi=$ $\left.-90^{\circ}\right)(<6 \mathrm{~Hz})$. As the $\mathrm{RH}$ increases to $90 \%$, all of those mechanisms shift toward a higher frequency side of the Bode plot because the ions are getting more mobile. A similar trend with humidity variation is also observed in DSLS samples as seen in Figure 3e. At $60 \% \mathrm{RH}$, at the low frequency range, the Bode plot shows that the DSLS sample form the electric double layer 18 times faster than LS, indicating the higher ionic conductivity of the DSLS compared to the LS sample. Indeed, the crossing between resistive and capacitive character represents the start of formation of the electric double layer at $\Phi=-45^{\circ}$, found at $7 \mathrm{~Hz}$ for LS and $125 \mathrm{~Hz}$ for DSLS. At 90\% RH, the double layer formation in DSLS is six times faster than LS. Total conductivity was calculated as $\sigma=d /(R A)$, where $d, A$, and $R$ are the distance between the electrodes, cross-sectional area of the drop-casted film, and the real part of impedance corresponding to the zero phase angle from the Bode plot, respectively. The total ionic conductivity of LS is $0.05 \mathrm{~S} / \mathrm{m}$ at $60 \% \mathrm{RH}$ and $8.2 \mathrm{~S} / \mathrm{m}$ at $90 \% \mathrm{RH}$, whereas the total conductivity of DSLS at $60 \% \mathrm{RH}$ is $0.73 \mathrm{~S} / \mathrm{m}$ and as high as $32 \mathrm{~S} / \mathrm{m}$ at $90 \% \mathrm{RH}$. A priori this is counterintuitive since the concentration of sulfonate groups in the layer governs the concentration of mobile cations in the layers. The conductivity is proportional to the product of the concentration of mobile ions and the mobility of the ions. Hence, since the DSLS has a lower concentration of sulfonate, it is expected to have a lower concentration of mobile cations; however, it has higher conductivity. But in our samples, we found out that DSLS also has 2.3 times more sulfate ions, thus introducing seven times higher concentrations in mobile ions $\left(\mathrm{H}^{+}, \mathrm{Na}^{+}, \mathrm{SO}_{4}{ }^{2-}\right)$. The difference in conductivity could also be due to a difference in morphology that will affect the mobility of ions in those layers. Indeed, LS is a plain layer, and the ions are transported within its bulk, while the DSLS layer is microporous where the pores are inactive in the ionic transport, except if they are filled with water at high humidity as found for other microporous sulfonated polyelectrolytes. ${ }^{21}$ Indeed, the measurements are performed at $90 \% \mathrm{RH}$, and both DSLS and LS are hygroscopic and wetted by water. Note that the KL layer, although less porous as seen by SEM, does not transport ions. Likely the pores in $\mathrm{KL}$ are not interconnected by percolation and have a closed porosity.

Choice of the Sulfonation Level for the Lignin/ Carbon Composite. While LS and DSLS layers show good ionic conductivity, the KL layer does not transport ions, which had already been suspected as a limiting phenomena for making thick lignin-carbon electrodes. ${ }^{15}$ Since none of those types of lignins transport electronic charge carriers, we are investigating now their composite with carbon black nanoparticles. This strategy is typically used to design battery electrodes. ${ }^{22}$ Carbon nanoparticles are interesting because they are small and have a large volume area, thus promoting large interface area with the redox insulating material; moreover, carbon is not covered by an insulating oxide and is certainly cheap. For the sake of comparison, the lignin/carbon composite electrodes were prepared by manually mixing the corresponding lignin (LS, DSLS, KL) and carbon black 

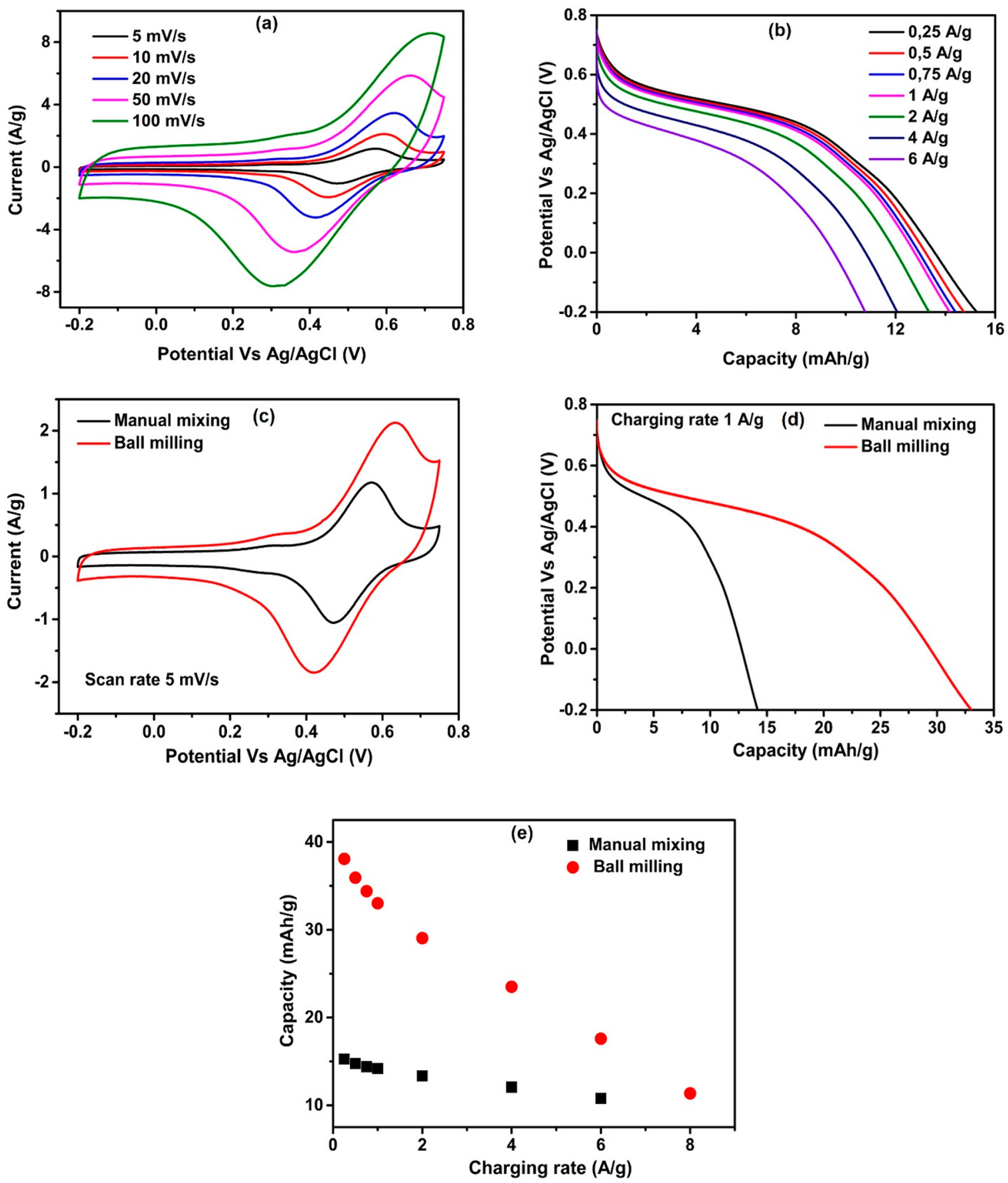

Figure 5. Panels (a) and (b) are the CV and the galvanostatic discharge plots of the manually mixed DSLS/C composites, (c-e) show the comparison of the manually mixed and ball milled DSLS/C composites in their CV, galvanostatic discharge, and the capacity versus the charging rate.

powders in a 1:1 weight ratio in water along with a binder system made of carboxymethyl cellulose (CMC) and styrenebutadiene rubber (SBR) (6 wt \%). This binder system has been optimized for graphite electrodes. ${ }^{23}$ In order to investigate the water stability of the lignin/carbon composite electrodes, they were blade-coated onto the carbon-coated stainless steel current collectors and dried at $60{ }^{\circ} \mathrm{C}$ for $15 \mathrm{~min}$. The dried electrodes were then dipped in water as shown in Figure $4 \mathrm{a}$, and the dissolution was examined by visual inspection. In the case of LS/C, the lignin leakage started within $30 \mathrm{~s}$ after dipping, followed by the dissolution of the composite electrode giving rise to a black color to water. This illustrates the challenge of using LS with carbon. Since DSLS and $\mathrm{KL}$ are not soluble in water, the DSLS/C and $\mathrm{KL} / \mathrm{C}$ composites are much more stable. The water used to dip the composite electrodes during $2 \mathrm{~h}$ was analyzed by UV spectroscopy (Figure 4b). The UV-vis spectrum reveals that lignin leakage exists in water from both LS/C and DSLS/C composites as evident by an absorption peak about $280 \mathrm{~nm}$ related to the aromatic monomeric rings of lignin. ${ }^{24-26}$ The samples were compared with the reference sample of aqueous solution of LS (shown as LS-std) in Figure $4 \mathrm{~b}$. The spectrum for the water analysis in which $\mathrm{KL} / \mathrm{C}$ was dipped does not display any specific optical transition due to lignin, but a clear 
background signal in the visible region attributed to the carbon nanoparticles. Such a signal in the visible region is also found for the LS/C composite that totally disintegrates in water. Hence, DSLS/C samples show the least disintegration/ dissolution of the entire electrode material along with carbon as compared to $\mathrm{LS} / \mathrm{C}$ and $\mathrm{KL} / \mathrm{C}$. DSLS is more efficient in forming a coherent composite with carbon and the binder compared to LS and KL. From the molecular weight analysis study (Figure 2b), it can be noted that there is a large difference in molecular weight for the DSLS and the starting material LS. A lot of the low molecular weight materials including sugars are washed away in the washing procedure after the neutralization of the lignin. Also, the DSLS contains a lot of high molecular weight lignin with MW of several 100 $\mathrm{kDa}$. The purpose with the chemical treatment of the lignin was to reduce solubility. This was achieved; however, the condensation of low MW lignin into insoluble high MW lignin is an important factor as the removal of sulfonic acid functionalities to reduce the leakage in water.

In order to study the electrical transport enabled by the carbon particles, impedance spectroscopy measurements of the lignin/carbon composites were carried out at $60 \% \mathrm{RH}$ and $90 \% \mathrm{RH}$. The Bode plot in Figure 4c shows a pure resistive behavior for $\mathrm{KL} / \mathrm{C}$ with a zero phase angle in the entire frequency region of $1 \mathrm{MHz}$ to $1 \mathrm{~Hz}$ at both relative humidity conditions. Hence, we believe that the impedance data of $\mathrm{KL} /$ $\mathrm{C}$ is solely representing the electronic carriers hopping between the carbon particles in the conducting percolation paths without any ionic contribution, as $\mathrm{KL}$ was ionically insulating. The total conductivity of $\mathrm{KL} / \mathrm{C}$ barely vary with humidity and is about $2.9-3 \mathrm{~S} / \mathrm{m}$. We observed a different behavior for LS/C and DSLS/C where the phase angle is not zero for the high frequency range (Figure $4 \mathrm{~d}, \mathrm{e}$ ), and thus does not behave as an ideal resistor anymore. Considering the behavior at $90 \% \mathrm{RH}$, we know that for the same electrode geometry, the pure LS film was displaying ionic transport already at $1 \mathrm{MHz}$ (Figure 3d) with values of ionic conductivity of about $8 \mathrm{~S} / \mathrm{m}$, which is the same order of magnitude as the electronic conductivity in the $\mathrm{KL} / \mathrm{C}$. Hence, we believe that ionic transport is occurring simultaneously with electronic transport in LS/C and DSLS/C composites. Our hypothesis is that the drop of the real part of the impedance toward high frequency is due to the screening of the electronic current passing through the percolation path of carbon particles by the ionic charges moving within the LS and DSLS surrounding matrix. We do not have enough data to attempt to distinguish between the ionic and electronic conductivity in those mixed electronic-ionic conductors, ${ }^{27,28}$ but we extract the total conductivity at a low frequency to be $5.4 \mathrm{~S} / \mathrm{m}(90 \% \mathrm{RH})$ for LS/C and $2.6 \mathrm{~S} / \mathrm{m}(90 \% \mathrm{RH})$ for DSLS/C. At $60 \% \mathrm{RH}$, the total conductivity from the low frequency region is $6.5 \mathrm{~S} / \mathrm{m}$ for LS/C and $2 \mathrm{~S} / \mathrm{m}$ for DSLS/C. The three composites have the same amount of carbon black and were prepared in identical manner, and hence the fact that they display the same order of magnitude for the total electrical conductivity indicates that in all those composites the carbon black particles are forming good electronic conducting percolation paths. KL/ $\mathrm{C}$ is mostly an electronic conductor, while LS/C and DSLS/C are mixed electron-ion conductors due to the physicochemical properties of the lignin that vary with the level of sulfonation. Because of the water stability of DSLS/C and its ionic conductivity, we thus focus the rest of this study on the optimization of the DSLS/C composites and its electrochemical characterization.

Processing Methods for the Fabrication DSLS-Carbon Electrodes. In order to investigate the significance of the intimate contact between the conducting carbon phase and the DSLS redox phase, the lignin/carbon composite was prepared either by manually mixing the components along with the binders or by using high energy ball milling. Figure 5a,b shows the cyclic voltammetry $(\mathrm{CV})(-0.2$ to $0.8 \mathrm{~V}$ vs $\mathrm{Ag} / \mathrm{AgCl})$ and galvanostatic discharge plots of the electrode in the potential range of -0.2 to $0.75 \mathrm{~V}$ vs $\mathrm{Ag} / \mathrm{AgCl}$, in $0.1 \mathrm{M} \mathrm{HClO}_{4}$. The composite electrode caste on to the carbon coated stainless steel substrate was used as the working electrode, and a $\mathrm{Pt}$ mesh was the counter electrode. The DSLS/C electrodes were electrochemically cycled using $\mathrm{CV}$ at different scan rates, namely, $100 \mathrm{mV} / \mathrm{s}, 50 \mathrm{mV} / \mathrm{s}, 20 \mathrm{mV} / \mathrm{s}, 10 \mathrm{mV} / \mathrm{s}$, and $5 \mathrm{mV} / \mathrm{s}$ (Figure 5a) and display two charge storage contributions: (i) A capacitive current contribution due to the formation of electric double layer at the surface of the carbon particles, which appears as a rectangular tail close to $0 \mathrm{~V}$. The capacitive current follows $I=-C \mathrm{~d} V / \mathrm{d} t$, where $C$ is the capacitance and $\mathrm{d} V / \mathrm{d} t$ is the scan rate. The current at $0 \mathrm{~V}$ increases indeed linearly with the scan rate, Figure S1. (ii) A Faradaic current appearing as a peak in the potential range of $0.4-0.65 \mathrm{~V}$, which corresponds to the proton-coupled electron transfer on the catechol/quinone groups of DSLS (chemical reaction in Figure $1)$. The current peak evolves linearly with the square root of the scan rate Figure S2, as indicative of a diffusion limited process likely due to the protons diffusion from the electrolyte to reach the lignin-carbon active interface. The galvanostatic charge-discharge measurements (Figure $5 b$ ) in the potential window of -0.2 to $0.75 \mathrm{~V}$, at various charging rates ranging from $0.25 \mathrm{~A} / \mathrm{g}$ up to $8 \mathrm{~A} / \mathrm{g}$, also displayed those two contributions. Discharge curves show a plateau in between the potential range of $0.45-0.6 \mathrm{~V}$, due to the Faradaic reactions of quinones, then a linear decay specific of a capacitive contribution between 0.4 to $-0.2 \mathrm{~V}$. The specific capacity of the manually mixed DSLS/C is $15 \mathrm{mAh} / \mathrm{g}$ at a $0.25 \mathrm{~A} / \mathrm{g}$ charging rate. We now compare the manual mixing with the process of making the composite by dry ball milling of DSLS and carbon black in planetary mill with $\mathrm{ZrO}_{2}$ milling container and balls. Note that the CMC/SBR binder was added after the active components are mixed either manually or by milling and the water-based slurry was formed. Figure $5 c-e$ shows the comparison of the manual mixed composite and the ball milled composite electrode in the $\mathrm{CV}$, galvanostatic discharge curve, and the capacity versus charging rate. The specific capacity of the DSLS/C composite prepared by ball milling $(32.5 \mathrm{mAh} / \mathrm{g}$ at $1 \mathrm{~A} / \mathrm{g})$ is more than twice that of the manual mixing (14 $\mathrm{mAh} / \mathrm{g}$ at $1 \mathrm{~A} / \mathrm{g}$ ), which is a clear indication that the milling process forms more redox active interface between the carbon conducting phase and the DSLS phase. The difference between the two processing methods to make the composite is that the ball milling is powerful enough to break the carbon black nanoparticles in smaller parts, ${ }^{29}$ thus, creating a larger lignin/C interface volume where electrons can reach the catechol redox site of the lignin (see Figure 1). We note however that the specific capacitance of the ball milled electrode drops dramatically with the charging rate, while the sample formed by manual mixing is almost constant. This suggests that the carbon percolation paths in the manually mixed DSLS/C composite are more electrically conducting than in the ball milled samples; the latter has difficulties to channel the 
electrical current from the large interfacial volume to the collector at a high charging rate. Despite that behavior, the ball milled DSLS/C is superior for the specific capacity in the whole range of charging rate and reaches the behavior of the manually mixed only for a very high charging rate. Hence, we thus focus now on the ball milling processing method for the electrodes and continue to optimize this electrode.

To understand the improved performance of the ball milled samples of DSLS/C, microstructural studies were carried out using SEM along with the energy dispersive X-ray spectroscopic analysis (EDX). The microstructure of the manually mixed sample (Figure 6a) consists of two distinct intermixed phases, namely, plate-like shapes (points 1 and 2) and fine spherical shapes (points 3 and 4). In contrast, the ball milled composite shows homogeneous morphology with grains of different dimensions (Figure 6b). Figure $6 c$ shows the compilation of the mass percentage of carbon and oxygen on different points on the surface for the above-mentioned composites. The EDX analysis performed on plate-like shapes (in Figure 6a points 1 and 2) shows a lower amount of carbon (86-81\%) and on the spherical morphology (in Figure 6a points 3 and 4) show a higher amount of carbon (94-95\%). The mass percentage of oxygen was higher for the plate-like shapes $(10-13 \%)$ as compared to spherical shapes $(2-4 \%)$. These observations indicate that the plate-like morphology corresponds to lignin particles, and the spherical shapes correspond to the carbon particles. The gray color contrast in the SEM image also reflects different atomic densities of the plate-like shape (lignin = light) compared to a spherical shape (carbon $=$ dark). In addition, this contrast variation suggests a difference in the conductivities of the two phases, with the lighter (lignin) phase less conducting than the darker (carbon) phase. However, these distinct microstructural features are not observed in the ball milled samples (Figure 6b); rather, they have a relatively homogeneous microstructure with grains of different sizes. Furthermore, the EDX analysis of different points on the sample surface shows a uniform distribution of carbon (88-90\%) and oxygen (6.9-8.5\%), suggesting the better intermixing of components at the nanometer scale during the ball milling process. The elemental mapping of the manually mixed and the ball milled samples is presented in the Figures S3 and S4.

Optimization of the Ball Milled DSLS-Carbon Electrodes. Further optimization of the composite relates to the ratio between the carbon conducting phase and the redox lignin phase in order to see the effect on the specific capacitance and its behavior versus charging rates (Figure $7 \mathrm{a}$ ). The ratio of DSLS/C was varied as $1: 4,1: 1$, and $3: 2$. The maximum capacity was obtained for a $1: 1$ composition. Further, we investigated the effect of the milling time (Figure $7 \mathrm{~b}$ ), from $2 \mathrm{~h}$ to $24 \mathrm{~h}$, by assuming that this will control the DSLS/C active interface volume in the electrode in which both electrons and protons can meet on the catechol redox site of lignin. For that purpose, we focused on the 1:1 composition of DSLS/C. Figure $7 \mathrm{~b}$ shows the galvanostatic discharge plot for electrodes with different durations of the milling, keeping the other milling parameters identical. The milling time was varied as 2 , $3,5,10$, and $24 \mathrm{~h}$. The electrode material shows improved performance as the milling time increases from 2 to $10 \mathrm{~h}$, due to the possible particle size reduction, leading to better nanoscale electrical contact leading to an improved charge transport between the conductor (carbon) and the lignin. There was an improvement of $15 \%$ in the capacity at $10 \mathrm{~h}$.
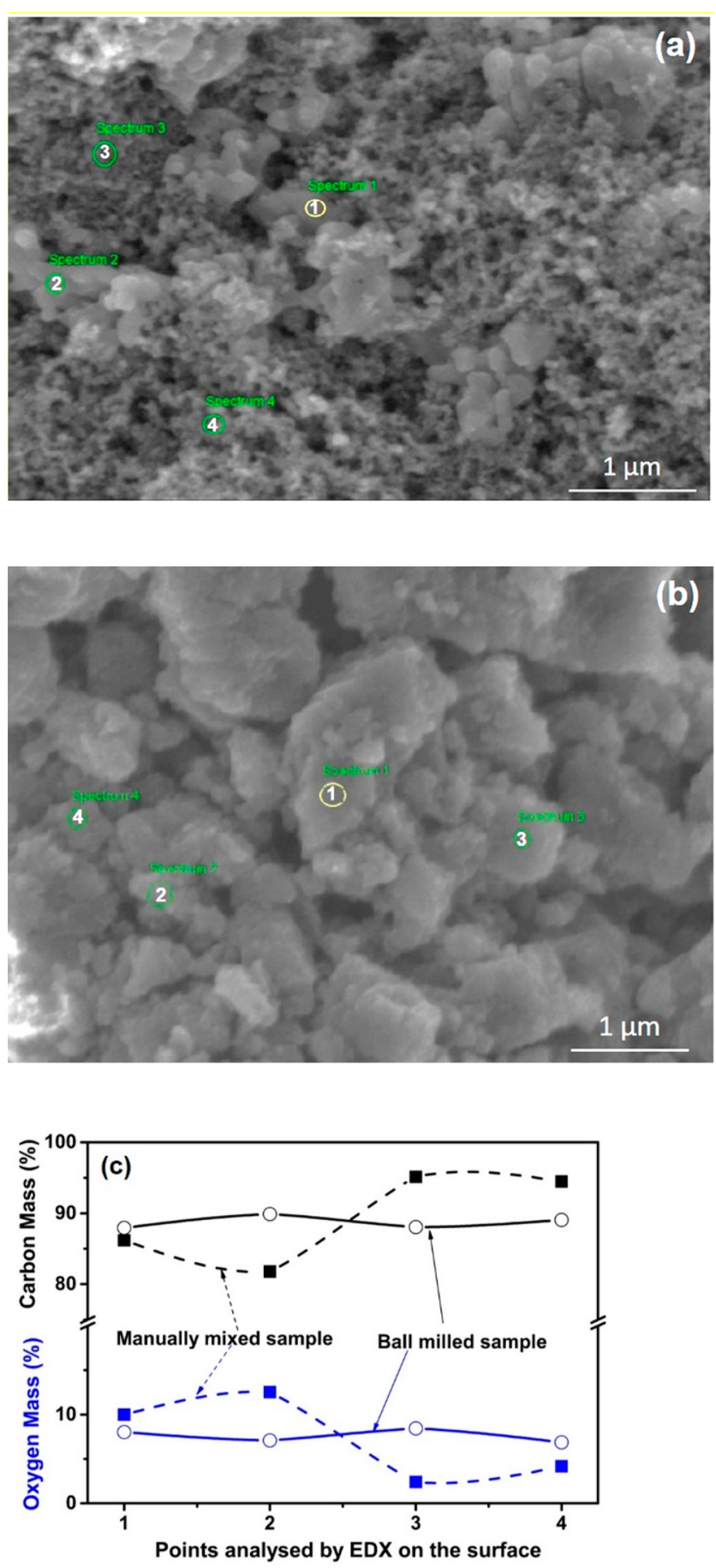

Figure 6. (a, b) SEM images of the manually mixed and ball milled DSLS/C composites respectively showing the regions analyzed by EDX. (c) The comparison of the carbon mass \% and oxygen mass \% at different regions on the surface for manually mixed and ball milled DSLS/C composites.

However, when the ball milling time was increased to $24 \mathrm{~h}$, the performance was found to be lower, indicating a trade off between the volume occupied by the conducting carbon percolation paths for the electronic carrier transport and the volume of accessible redox site in the DSLS phase. The degradation of the performance above $10 \mathrm{~h}$ ball milling suggests a possible chemical degradation of lignin due to heat generation during the milling process. We also investigated the effect of the type of carbon material, namely, the carbon black 

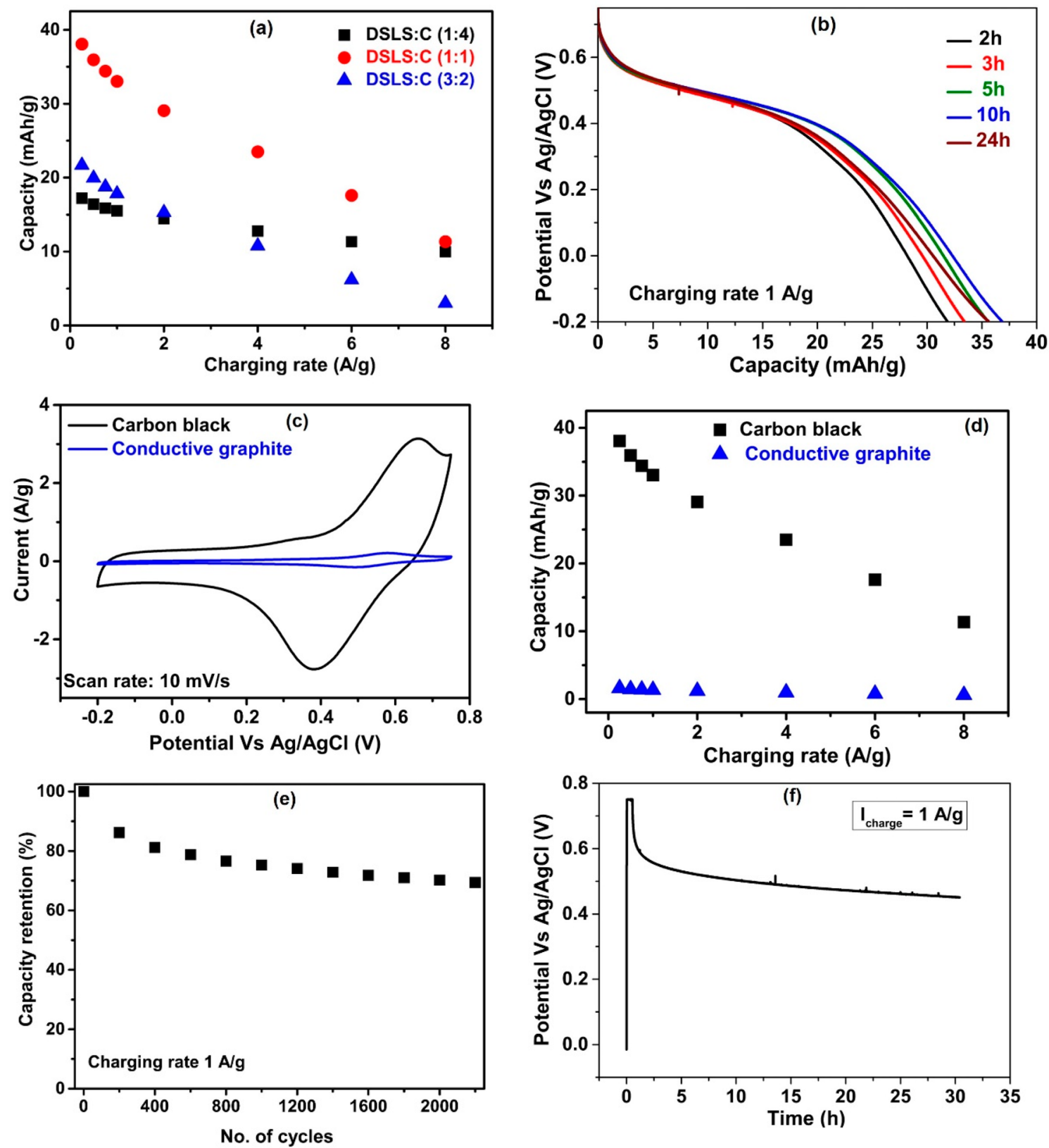

Figure 7. (a) Capacity vs charging rates for composite electrodes with different ratios of carbon and DSLS, (b) the galvanostatic discharge plot at 1 $\mathrm{A} / \mathrm{g}$ charging rate for DSLS/C (1:1) samples milled for different durations, (c) and (d) show the cyclic voltammetry at $10 \mathrm{mV} / \mathrm{s}$ scan rate, the capacity versus charging rate for DS-LS/C (1:1) sample, where the carbon type was varied, (e) galvanostatic charge-discharge stability (cycle) of the DSLS/C electrode of composition of $1: 1$ with a charging rate of $1 \mathrm{~A} / \mathrm{g}$ in ambient condition and (f) self-discharge study of the electrode in 0.1 $\mathrm{M} \mathrm{HClO}_{4}$ in nitrogen atmosphere.

and the conductive graphite. Here the intension was to understand the impact of the nature of carbon on the electrochemical behavior even after ball milling. And the effect is striking as presented in Figure $7 \mathrm{c}, \mathrm{d}$. The DSLS/conducting carbon has a higher specific capacitance $(32 \mathrm{mAh} / \mathrm{g}$ at $1 \mathrm{~A} / \mathrm{g})$ compared to the corresponding composite with graphite (2 $\mathrm{mAh} / \mathrm{g}$ at $1 \mathrm{~A} / \mathrm{g}$ ) for a similar DSLS/C ratio. This is somehow a surprise since a recent study using ball milling with LS and graphite (with 1:1 ratio) reached a discharge capacity of 25 $\mathrm{mAh} / \mathrm{g}$ at $1 \mathrm{~A} / \mathrm{g} .{ }^{14}$ The DSLS used in the present study does not seem to be efficient in shearing the graphite as compared to the LS in the above-reported study. Importantly, that study reveals a clear leakage of LS in aqueous electrolytes under prolonged electrochemical cycles, mostly in acidic electrolytes, leading to a $40 \%$ drop in specific capacity within 1000 cycles. In order to investigate the electrochemical stability, the DSLS/ $\mathrm{C}$ electrode was studied in $0.1 \mathrm{M} \mathrm{HClO}_{4}$ electrolyte in a threeelectrode method by galvanostatic charge/discharge (Figure $7 \mathrm{e})$ at ambient condition. The charging rate used was $1 \mathrm{~A} / \mathrm{g}$, and the potential range was from -0.2 to $0.75 \mathrm{~V}$ versus $\mathrm{Ag} /$ $\mathrm{AgCl}$. A $40 \%$ reduction in the specific capacitance was observed after 2200 cycles. Self-discharge was measured in nitrogen atmosphere with a charging rate of $1 \mathrm{~A} / \mathrm{g}$ during the charging stage. The electrode was charged to a potential of $0.75 \mathrm{~V}$ vs $\mathrm{Ag} / \mathrm{AgCl}$ and kept at that potential for 30-60 min, followed by an open circuit measurement. The data are shown in Figure $7 f$, where the potential dropped by $40 \%$ of the initial value after $30 \mathrm{~h}$. 


\section{DISCUSSION}

We provide an overview of the total specific capacity of various lignin-based electrodes found in the literature versus the percentage of charge retention after 100 cycles (Figure 8a). This is an attempt to compare the energy storage ability and the stability. We observe two groups of data, one around 70$80 \mathrm{mAh} / \mathrm{g}$ for LS/PAAQ/PEDOT, ${ }^{9} \mathrm{KL} / \mathrm{C},{ }^{15,16} \mathrm{LS} / \mathrm{RGO}^{11}$ and one group around $30-40 \mathrm{mAh} / \mathrm{g}$ for LS/graphite, ${ }^{14} \mathrm{KL} /$ $\mathrm{CNT}^{12}{ }^{12}$ LS/PEDOT, ${ }^{6}$ and LS-PEDOT:PSS. ${ }^{18}$ As mentioned in the introduction, a positively charged conducting polymer complexed with negatively charged LS provides a stable electrode (e.g., LS-PEDOT), but LS combined with graphite leads to a less stable electrode because of the solubility of LS in the electrolyte (LS-graphite). The reduced graphene oxide, RGO, seems to provide better stability possibly due to specific interaction between LS and the polar groups of RGO. DSLS improves the stability of the electrode composited with carbon. Our DSLS/C electrode is in the group with a low specific energy, but when we examine a bit closer our DSLS/C electrode with the competitive electrodes based on $\mathrm{KL} / \mathrm{C}$, ${ }^{15}$ we observe a striking difference important to underline in a discussion. Indeed, for the sake of comparison between our work and the high specific capacity of $\mathrm{KL} / \mathrm{C},{ }^{15}$ the capacity was calculated from the $\mathrm{CV}$ curve measured at a scan rate of 5 $\mathrm{mV} / \mathrm{s}$ in $0.1 \mathrm{M} \mathrm{HClO}_{4}$. The total capacity calculated from the $\mathrm{CV}$ curve has two contributions, one from the capacitive effect (non-Faradaic contribution) due to the formation of electric double layer at the carbon surface, and the other originating from the redox processes (Faradaic contribution) of lignin as shown in Figure $8 \mathrm{~b}$. Figure $8 \mathrm{c}$ shows the comparison of the DSLS/C composite prepared by ball milling with the $\mathrm{KL} / \mathrm{C}$ electrode $^{15}$ also prepared via ball milling. Besides the differences in the milling method used, it is also important to note that the DSLS/C slurry is completely water based (binder CMC/SBR), unlike the $\mathrm{KL} / \mathrm{C}$ using PVDF binder in $\mathrm{N}$-methyl pyrrolidone. In our DSLS/C electrode, $66 \%$ of the total charge storage capacity is due to the contribution from the Faradaic process of lignin, whereas for the KL/C electrode only $11 \%$ of the specific capacity comes from the lignin redox activity (Figure 8c). Thus, the large specific capacitance of KL/ $\mathrm{C}$ is mostly due to the good quality and high surface area of the carbon nanoparticles in the composite. This indicates that the accessing of the redox sites is more effective in our approach due to a high interfacial volume in DSLS/C where both electrons and ions can be accessed. We believe that this is due to the good ionic conductivity of DSLS compared to KL. The result of this strong Faradaic contribution is a discharge curve with a clear plateau in the range $(0.4-0.6 \mathrm{~V}$ ) (Figure 8d), while other studies for $\mathrm{KL} / \mathrm{C}$ display a rather linear decay with a weak shoulder. This conclusion is further evidenced by the recent report of LS/graphite processed by ball milling ${ }^{14}$ and the LS/RGO displaying both a strong Faradaic contribution from LS due to its ionic conductivity compared to KL.

\section{CONCLUSIONS}

We demonstrate that the desulfonation of LS by simple alkali treatment takes place at the same time as the formation of high molecular weight fractions due to condensation. The resulting DSLS is insoluble in water, but the nanoparticles formed are easily wetted by water to make a water-processable DSLS slurry that is perfect for large-scale coating in industrial premises. Interestingly, the ionic conductivity of DSLS is
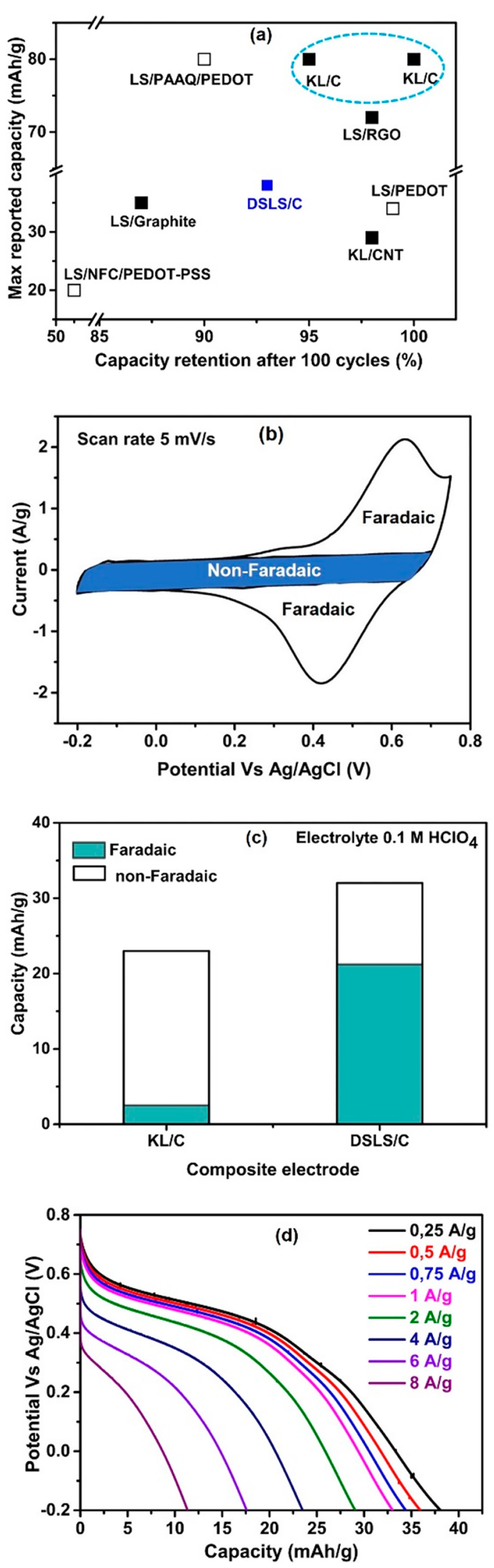

Figure 8. (a) Maximum capacity reported of lignin-based electrodes versus the percentage of capacity retention after 100 cycles for various studies found in the literature: LS/graphite, ${ }^{14}$ LS/PAAQ/PEDOT, ${ }^{9}$ $\mathrm{KL} / \mathrm{C},{ }^{15,16} \mathrm{KL} / \mathrm{CNT},{ }^{12} \mathrm{LS} / \mathrm{RGO}^{11}{ }^{11}$ and LS/PEDOT. ${ }^{6}$ Conducting polymers composites are represented by open square symbol, and the 
Figure 8. continued

composites with other carbon materials are shown with filled square symbol. The data from the present study are shown in blue filled square symbol. (b) CV of the DSLS-C electrode having a 1:1 composition along with $6 \mathrm{wt} \%$ binder. The measurement was done in $0.1 \mathrm{M} \mathrm{HClO}_{4}$ at a scan rate of $5 \mathrm{mV} / \mathrm{s}$. (c) Comparison of the total capacity and deconvoluted contributions (non-Faradaic from carbon and Faradaic from lignin with the literature data for the points related to $\mathrm{KL} / \mathrm{C}) .{ }^{15}$ (d) The discharge plots at different charging rates for the DSLS-C composite.

higher than LS, while $\mathrm{KL}$ is nonconducting. The combination of good ionic conductivity and insolubility make DSLS unique compared to LS and KL and ideal for energy storage applications. The effectiveness of the ball milling process to form the composite of DSLS/C leads to a stable electrode that displays a high Faradaic contribution to the total specific capacitance, thus making it unique compared to $\mathrm{KL} / \mathrm{C}$ electrodes. This study brings us one step further towards the design of efficient lignin-based electrodes for large-scale nonflammable energy storage solutions.

\section{ASSOCIATED CONTENT}

\section{(s) Supporting Information}

The Supporting Information is available free of charge at https://pubs.acs.org/doi/10.1021/acssuschemeng.0c05397.

Current (at $0 \mathrm{~V}$ ) versus scan rate, current (at redox potential) versus $\sqrt{ }$ scan rate for manually mixed DSLS/ $\mathrm{C}$, EDX elemental mapping of manually mixed and ball milled DSLS/C (PDF)

\section{AUTHOR INFORMATION}

\section{Corresponding Author}

Xavier Crispin - Laboratory of Organic Electronics, Bredgatan 33, 60174, ITN and Wallenberg Wood Science Center, Bredgatan 33, 60174, ITN, Linköping University, Norrköping, Sweden; 이이.org/0000-0001-8845-6296; Email: xavier.crispin@liu.se

\section{Authors}

Ujwala Ail - Laboratory of Organic Electronics, Bredgatan 33, 60174, ITN, Linköping University, Norrköping, Sweden

Jaywant Phopase - Laboratory of Organic Electronics, Bredgatan 33, 60174, ITN, Linköping University, Norrköping, Sweden; (1) orcid.org/0000-0002-7179-6670

Jakob Nilsson - Ligna Energy AB, 60174 Norrköping, Sweden

Zia Ullah Khan - Laboratory of Organic Electronics, Bredgatan 33, 60174, ITN, Linköping University, Norrköping, Sweden

Olle Inganäs - Department of Physics, Chemistry and Biology IFM, Linkopings Universitet, Linköping, Sweden 58183; (1) orcid.org/0000-0002-6243-1450

Magnus Berggren - Laboratory of Organic Electronics, Bredgatan 33, 60174, ITN and Wallenberg Wood Science Center, Bredgatan 33, 60174, ITN, Linköping University, Norrköping, Sweden

Complete contact information is available at:

https://pubs.acs.org/10.1021/acssuschemeng.0c05397

\section{Notes}

The authors declare no competing financial interest.

\section{ACKNOWLEDGMENTS}

The authors thank the Knut and Alice Wallenberg Foundation (proof of concept "Paper battery", Wallenberg Wood Science Center, a Wallenberg Scholar (O.I.)) and Vinnova (Treesearch, Digital Cellulose Center), Advanced Functional Materials Center at Linköping University. The authors thank Peter Ringstad working at Ligna Energy $A B$ for discussions and project management, Henrik Wallmo working at Valmet $A B$ for discussions and providing the KL samples, Edblad Magnus working at Domsjö-Adityabirla for discussions, chemical analysis, and providing the LS samples.

\section{REFERENCES}

(1) Barnes, F., Levine, J., Ed. Large Energy Storage Systems Handbook; CRC Press: Boca Raton, 2011, DOI: 10.1201/b10778.

(2) Liedel, C. Sustainable Battery Materials from Biomass. ChemSusChem 2020, 13, 2110-2141.

(3) National Research Council. The Role of the Chemical Sciences in Finding Alternatives to Critical Resources: A Workshop Summary. The National Academic Press: Washington, DC, 2012. DOI: 10.17226/ 13366.

(4) Espinoza-Acosta, J. L.; Torres-Chávez, P. I.; Olmedo-Martínez, J. L.; Vega-Rios, A.; Flores-Gallardo, S.; Zaragoza-Contreras, E. A. Lignin in storage and renewable energy applications: A review. J. Energy Chem. 2018, 27, 1422-1438.

(5) Aro, T.; Fatehi, P. Production and Application of Lignosulfonates and Sulfonated Lignin. ChemSusChem 2017, 10, 1861-1877.

(6) Ajjan, F. N.; Casado, N.; Rebis, T.; Elfwing, A.; Solin, N.; Mecerreyes, D.; Inganas, O. High performance PEDOT/lignin biopolymer composites for electrochemical supercapacitors. J. Mater. Chem. A 2016, 4, 1838-1847.

(7) Milczarek, G.; Inganas, O. Renewable Cathode Materials from Biopolymer/Conjugated Polymer Interpenetrating Networks. Science 2012, 335, 1468-1471.

(8) Ajjan, F. N.; Jafari, M. J.; Rebis, T.; Ederth, T.; Inganas, O. Spectroelectrochemical investigation of redox states in a polypyrrole/ lignin composite electrode material. J. Mater. Chem. A 2015, 3, 12927-12937.

(9) Ajjan, F. N.; Vagin, M.; Rebis, T.; Aguirre, L. E.; Ouyang, L.; Inganas, O. Scalable Asymmetric Supercapacitors Based on Hybrid Organic/Biopolymer Electrodes. Adv. Sustainable Syst. 2017, 1, 1700054.

(10) Geng, X.; Zhang, Y.; Jiao, L.; Yang, L.; Hamel, J.; Giummarella, N.; Henriksson, G.; Zhang, L.; Zhu, H. Bioinspired Ultrastable Lignin Cathode via Graphene Reconfiguration for Energy Storage. ACS Sustainable Chem. Eng. 2017, 5, 3553-3561.

(11) Kim, S. K.; Kim, Y. K.; Lee, H.; Lee, S. B.; Park, H. S. Superior Pseudocapacitive Behavior of Confined Lignin Nanocrystals for Renewable Energy-Storage Materials. ChemSusChem 2014, 7, 10941101.

(12) Milczarek, G.; Nowicki, M. Carbon nanotubes/kraft lignin composite: Characterization and charge storage properties. Mater. Res. Bull. 2013, 48, 4032-4038.

(13) Navarro-Suarez, A. M.; Casado, N.; Carretero-Gonźalez, J.; Mecerreyes, D.; Rojo, T. Full-cell quinone/hydroquinone supercapacitors based on partially reduced graphite oxide and lignin/ PEDOT electrodes. J. Mater. Chem. A 2017, 5, 7137-7143.

(14) Liu, L.; Solin, N.; Inganas, O. Scalable lignin/graphite electrodes formed by mechanochemistry. RSC Adv. 2019, 9, 39758-39767.

(15) Chaleawlert-umpon, S.; Berthold, T.; Wang, X.; Antonietti, M.; Liedel, C. Kraft Lignin as Electrode Material for Sustainable Electrochemical Energy Storage. Adv. Mater. Interfaces 2017, 4, 1700698 .

(16) Chaleawlert-umpon, S.; Liedel, C. More sustainable energy storage: lignin based electrodes with glyoxal crosslinking. J. Mater. Chem. A 2017, 5, 24344-24352. 
(17) Che, C.; Vagin, M.; Ail, U.; Gueskine, G.; Phopase, J.; Brooke, R.; Gabrielsson, R.; Jonsson, M. P.; Mak, W. C.; Berggren, M.; Crispin, X. Twinning Lignosulfonate with a Conducting Polymer via Counter-Ion Exchange for Large-Scale Electrical Storage. Adv. Sustainable Syst. 2019, 3, 1900039.

(18) Edberg, J.; Inganas, O.; Engquist, I.; Berggren, M. Boosting the capacity of all-organic paper supercapacitors using wood derivatives. $J$. Mater. Chem. A 2018, 6, 145-152.

(19) Larsson, O.; Said, E.; Berggren, M.; Crispin, X. Insulator Polarization Mechanisms in Polyelectrolyte-Gated Organic FieldEffect Transistors. Adv. Funct. Mater. 2009, 19, 3334-3341.

(20) Wang, H.; Zhao, D.; Khan, Z. U.; Puzinas, S.; Jonsson, M. P.; Berggren, M.; Crispin, X. Ionic Thermoelectric Figure of Merit for Charging of Supercapacitors. Adv. Electron. Mater. 2017, 3, 1700013.

(21) Jiao, F.; Edberg, J.; Zhao, D.; Puzinas, S.; Khan, Z. U.; Mäkie, P.; Naderi, A.; Lindström, T.; Odén, M.; Engquist, I.; Berggren, M.; Crispin, X. Nanofibrillated Cellulose-Based Electrolyte and Electrode for Paper-Based Supercapacitors. Adv. Sustainable Syst. 2018, 2, 1700121.

(22) Kwon, N. H.; Mouck-Makanda, D.; Fromm, K. M. A Review: Carbon Additives in $\mathrm{LiMnPO}_{4}-$ and $\mathrm{LiCoO}_{2}$-Based Cathode Composites for Lithium Ion Batteries. Batteries 2018, 4, 50.

(23) Chang, W. J.; Lee, G. H.; Cheon, Y. J.; Kim, J. T.; Lee, S. I.; Kim, J.; Kim, M.; Park, W. I.; Lee, Y. J. Direct Observation of Carboxymethyl Cellulose and Styrene-Butadiene Rubber Binder Distribution in Practical Graphite Anodes for Li-Ion Batteries. ACS Appl. Mater. Interfaces 2019, 11 (44), 41330-41337.

(24) Li, Z.; Ge, Y. Extraction of lignin from sugar cane bagasse and its modification into a high performance dispersant for pesticide formulations. J. Braz. Chem. Soc. 2011, 22, 1866-1871.

(25) Heitner, C., Dimmel, D. R, Schmidt, J. A, Ed. Lignin and Lignans: Advances in Chemistry; CRC Press Taylor \& Francis Group, 2010, DOI: 10.1201/EBK1574444865.

(26) Fergus, B. J.; Goring, D. A. I. The Distribution of Lignin in Birch Wood as Determined by Ultraviolet Microscopy. Holzforschung 1970, 24, 118.

(27) Ail, U.; Jafari, M. J.; Wang, H.; Ederth, T.; Berggren, M.; Crispin, X. Thermoelectric Properties of Polymeric Mixed Conductors. Adv. Funct. Mater. 2016, 26, 6288-6296.

(28) Lai, W.; Haile, S. M. Impedance Spectroscopy as a Tool for Chemical and Electrochemical Analysis of Mixed Conductors: A Case Study of Ceria. J. Am. Ceram. Soc. 2005, 88, 2979-2997.

(29) Hasan, M. H. M.; Ramli, H.; Ong, S. K.; Manroshan, S. Study on the effect of ball milling time towards the stability of carbon black dispersion and carbon black masterbatch properties. AIP Conference Proceedings 1985, 040014, 2018, DOI: DOI: 10.1063/1.5047191. 